\title{
«2\% de réduction sur tous les achats pour les nouveaux clients Galexis»
}

\section{Félicitations à I'heureux gagnant!}

Le week-end Bien-être pour deux au

\section{Parkhotel Delta $\star \star \star \star \star$ Ascona}

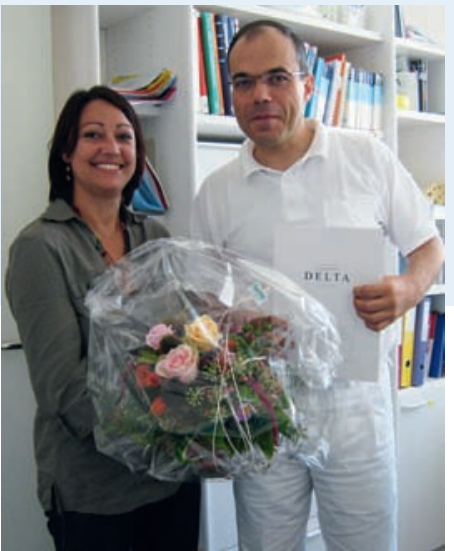

a été remporté par le Dr Thomas Clerc, Aadorf (à droite sur la photo). Madame Sandra Stadelmann de FMH Consulting Services (à gauche sur la photo) remet son prix à l'heureux gagnant.

Ce prix est sponsorisé par le Parkhotel Delta Ascona.

Vous aimeriez également régler vos achats avec le système de factures groupées de FMH Consulting Services? Tous les nouveaux clients bénéficient pendant un an d'une réduction de $2 \%$ sur les achats effectués chez nos fournisseurs contractuels.

Les avantages sont les suivants:

- facture mensuelle unique regroupant tous les achats effectués auprès des fournisseurs contractuels sur une période de 4 à 6 semaines;

- le nombre de vos opérations de comptabilité se limite à un seul paiement;

- moins de frais bancaires;

- nos fournisseurs contractuels sélectionnés avec soin sont la garantie de prestations de service de qualité et de conditions avantageuses.
Voici comment cela fonctionne:

- vous commandez directement auprès de nos fournisseurs contractuels;

- ceux-ci envoient à FMH Consulting Services un décompte mensuel;

- FMH Consulting Services règle le décompte le 20 du mois suivant;

- le 15 du mois, vous recevez une facture groupée.

\section{Coupon-réponse}

Merci d'envoyer ou de faxer ce coupon au : 0419210586

Nom/Prénom

Adresse

Case postale/Localité

Tél. privé

Tél. bureau

Numéro RCC (numéro de concordat)

E-mail

Localité/Date/Signature

Je souhaite à l'avenir faire mes achats auprès des fournisseurs suivants et en régler le montant via le système de factures groupées. J'ai pris connaissance du fait que le service de factures groupées proposé par FMH Consulting Services m'est offert gratuitement.

Aichele Medico AG, Aesch

Apotheke zum Mörser, Zurich

Dr. F. Rappai, Schlieren

Galexis AG, Schönbühl

IVF, Neuhausen

O Lohmann \& Rauscher AG, Flawil

Medicare AG, Zurich

Mepha Pharma AG, Aesch BL

Polymed Medical Center, Glattbrugg

Raetus-Apotheke, Coire

Rufma AG, Bremgarten
Salzmann AG, St-Gall

Spirig AG, Egerkingen

Spiromed, Gelterkinden

O Streuli Pharma AG, Uznach

Uneltech Electronic AG, Schwerzenbach

O Unitobler Apotheke, Berne 